ORIGINAL ARTICLE

\title{
Effects of clinical service reorganisation on cellular pathology workload
}

\author{
S Winter, G J Cox, R Corbridge, A J Chaplin, P R Millard, K A Shah
}

See end of article for authors' affiliations

.....................

Correspondence to: Dr K A Shah, Department of Cellular Pathology, The John Radcliffe Hospital, Headington, Oxford OX3 9DU, UK; ketan. shah@orh.nhs.uk

Accepted for publication 9 July 2003

\begin{abstract}
Aims: To assess changes in volume and complexity of cellular pathology workload after clinical service reorganisation and alterations in pathology reporting practices, and to identify objective measures of change applicable to all cellular pathology departments. The ear, nose, and throat (ENT), head and neck (HN) specialty was chosen for assessment.

Methods: Cellular pathology workload from the ENT-HN surgical specialty was assessed numerically and the complexity in examination of cancer resection specimens was evaluated. Medical and technical time inputs in the reporting of ENT-HN cancer resections were measured prospectively, and the histological and cytological workload arising from the management of such cases was obtained.

Results: The $88.83 \%$ increase in ENT-HN specimens contrasted with a $13.53 \%$ increase in total surgical workload. Substantial increases in work complexity were found when measured as blocks/slides for each case and number of histochemical/immunohistochemical requests. On average, examination of one ENT$\mathrm{HN}$ cancer case consumed 55\% of one pathologist's work session and over one 10th of a technician's working week. On average, each cancer generated 3.3 histological and 1.06 cytological specimens. Conclusions: Evidence is provided of the increase in cellular pathology workload and in its complexity. This study lists objective measures of complexity applicable to all pathology subspecialties. Given the workforce crisis and expanding clinical needs, realistic workload calculations should include measurement of complexity and not just volumes.
\end{abstract}

"Although numerical increases are easy to measure, the complexity of pathological analysis is more difficult to quantify"

One of the key recommendations of the Calman-Hine report was that patients with cancer should be treated in regional cancer centres. ${ }^{1}$ A multidisciplinary approach was advocated in this report, which has been widely accepted as the current model by those professional associations with an interest in the field. ${ }^{23}$ The currently awaited NICE (National Institute for Clinical Excellence) guidelines for head and neck (HN) cancer are expected to concur with and expand upon this centralist model. Based on the favoured model of concentration of expertise in fewer centres, the HN oncology service at the Oxford Radcliffe Hospitals NHS Trust was developed in 1995 within the ear, nose, and throat (ENT) department. Not only has this evolved into a cancer centre as defined by the National Health Service cancer plan, ${ }^{4}$ but it is also the regional tertiary referral centre. It serves a population of 850000 and has the services of three head and neck surgeons. This reorganisation has directly affected the cellular pathology department of the trust.

In tandem, the cellular pathology workload has changed as a result of the publication of minimum dataset guidelines for reporting of cancer specimens, and because of the expanding definition of disease subsets and new entities using immunohistochemical and molecular biological techniques. Although numerical increases are easy to measure, the complexity of pathological analysis is more difficult to quantify.

With these factors in mind, a retrospective and part prospective study was carried out to measure the alteration in cellular pathology workload, not only by defining the quantitative change, but by also evaluating the complexity of the analysis. In addition, the impact on cellular pathology of the management of patients with cancer within the ENT-HN subspecialty was sought from the number of histological and cytological investigations undertaken during their care. The aim was to document and provide an objective measure of change applicable to all cellular pathology departments.

\section{MATERIALS AND METHODS}

Our numerical study was based on an analysis of specimens received in the cellular pathology laboratory of the John Radcliffe Hospital, which serves the HN cancer centre. The data were collected from the departmental computer records and anonymised so as not to compromise patient identity and/or confidentiality.

The number of histological specimens from the ENT-HN specialty was compared with the total number of surgical specimens received between January 1995 and December 2001. Each entry corresponded to specimen(s) received from a single patient at any one point, regardless of their number. The start period coincided with clinical service reconfiguration in 1995. Within these figures, the number of "large" specimens from the resection of ENT-HN cancer was identified. These included resections of tongue, tonsil, larynx,

Abbreviations: ENT, ear, nose and throat; FNA, fine needle aspiration; $\mathrm{HN}$, head and neck 
pharynx, and neck dissections, singly or in varying combinations. Specimens of maxillofacial and haematological malignancies were excluded from the analysis. The data on the number of patients referred to the ENT department were available from financial year 1997/98 and were obtained up to 2000/2001. These were the closest comparable data on hand for the cellular pathology calendar workload figures.

There are no currently accepted and standard measures of the complexity of pathological examination. Complicated specimens increase the workload for both pathologists and technical laboratory staff, in terms of the number of routine and/or additional studies carried out. Thus, one indicator of the complexity could be the number of paraffin wax blocks and slides examined. These were obtained for all ENT-HN cases, and from these figures the data for cancer resection specimens were distilled. Another measure is the time taken for the analytical process. Although this can be computed for both small biopsies and large resection specimens, it is the larger specimens that have been greatly affected by minimum dataset guidelines. In our study, a prospective analysis of 11 consecutive large cancer cases was undertaken to obtain the time and number of blocks taken for their examination. The pathologists' input was calculated in terms of prosection time, microscopy time, and report authorisation (including dataset generation) time. The technical input was calculated as Welcan units for each case, to include all laboratory activities from specimen reception to the generation of slides. ${ }^{5}$ Finally, the need for histochemical and immunohistochemical stains indicates the evaluation of processes not identified on routine haematoxylin and eosin stained sections, and thus reflects complexity. Their numbers were obtained for all ENT-HN cases. All of the above data were collected between January 1998 and December 2001. The publication in 1998 of the minimum dataset for reporting $\mathrm{HN}$ cancer specimens by the Royal College of Pathologists provided a benchmark for the measure of complexity. ${ }^{6}$

To obtain an estimate of the work generated for cellular pathology during the management of ENT-HN cancer, a retrospective analysis of 15 randomly selected patients who underwent cancer resections in 2001 was carried out. All

Table 1 Histological workload showing total surgical cases (1995-2001), ENT-HN specimens (1995-2001), and ENT-HN cancer specimens (1998-2001)

\begin{tabular}{llll}
\hline Year & Total cases & ENT-HN cases & $\begin{array}{l}\text { ENT-HN cancer } \\
\text { cases }\end{array}$ \\
\hline 1995 & 23996 & $439(1.82 \%)$ & Not available \\
1996 & 26083 & $702(2.69 \%)$ & Not available \\
1997 & 25670 & $704(2.74 \%)$ & Not available \\
1998 & 26214 & $607(2.31 \%)$ & $55(9.06 \%)$ \\
1999 & 26521 & $756(2.85 \%)$ & $53(7.01 \%)$ \\
2000 & 25842 & $731(2.83 \%)$ & $65(8.89 \%)$ \\
2001 & 27244 & $829(3.04 \%)$ & $62(7.47 \%)$ \\
\hline
\end{tabular}

The figures in parenthesis in the ENT-HN cases column are a percentage of all cases for that year. The figures in parenthesis in the ENT-HN cancer cases column are a percentage of ENT-HN cases for that year.

ENT, ear, nose, and throat; $\mathrm{HN}$, head and neck. histological and cytological investigations from diagnosis to those in the follow up period were recorded. When a patient was investigated at another regional hospital, its pathology department was contacted to find out the number of tests performed. All data were subsequently processed anonymously, without compromising patient identity and/or confidentiality.

\section{RESULTS}

Table 1 presents an analysis of the histological workload (1995 and 2001) divided into total surgical biopsies, all ENTHN cases, and ENT-HN cancer resections. In 1995, ENT-HN cases accounted for $1.81 \%$ of the total workload, and this number had increased to $3.04 \%$ by 2001 . In that period, although the overall surgical pathology workload increased by $13.53 \%$, the number of ENT-HN specimens increased by 88.83\% (from 439 to 829). From 1998 to 2001, there was a slight increase in the number of ENT-HN cancer resections ( 55 to 62 ), although as a proportion of all ENT-HN cases, this fell from $9.06 \%$ to $7.47 \%$.

In the financial year 1997/98, 6604 patients were referred to the ENT department, and this number had increased to 7409 (by 12.18\%) by 2000/2001. Between 1998 and 2001, the ENT-HN cellular pathology workload increased by $36.57 \%$, from 607 to 829 cases.

Table 2 presents the data on the number of paraffin wax blocks (including oversize blocks), slides, special stain requests, and immunohistochemical examinations between 1998 and 2001. During this period, a 36.57\% increase in all ENT-HN specimens was reflected by increases of $57 \%$ and $63.7 \%$ in the number of paraffin wax blocks and slides examined, respectively. The number of ENT-HN cancer resections increased from $55 \%$ to $62 \%(12.72 \%)$ between 1998 and 2001, whereas the number of blocks and slides examined for these increased by $70 \%$ and $73 \%$, respectively. When calculated as the number of blocks and slides examined for each case of ENT-HN cancer resection, this increased by $50.9 \%$ (10.9 to 16.45 ) and $53 \%$ ( 11.18 to 17.16 ), respectively. The number of oversize blocks examined for these cases nearly doubled in this period. When cancer resection specimens were excluded from the analysis, the increase in the number of blocks and slides for all other ENTHN cases was a more modest $8 \%$ (2.05 to 2.22) and 14\% (2.35 to 2.69 ), respectively.

Table 3 presents the results of the prospective time analysis study of 11 consecutive cancer resection cases. The specimens included resections of the tongue, tonsil, larynx, pharynx, and neck dissection, individually or in varying combinations. The pathologist's time input ranged from 39 minutes for a unilateral neck dissection specimen to 220 minutes for a tongue resection with bilateral neck dissections. Of the 11 specimens, three consisted of neck dissections alone, with time inputs of 39, 45, and 95 minutes (average, 60 minutes). The other eight specimens comprised resections of primary tumour along with unilateral or bilateral neck dissection. The reporting time on these ranged from 65 to 220 minutes (average, 140 minutes). When considered all together, the complete examination of one cancer resection specimen took

Table 2 Laboratory data for ENT, head and neck specimens, 1998-2001

\begin{tabular}{|c|c|c|c|c|c|c|c|}
\hline Year & $\begin{array}{l}\text { All ENT-HN } \\
\text { blocks }\end{array}$ & $\begin{array}{l}\text { All ENT-HN } \\
\text { slides }\end{array}$ & $\begin{array}{l}\text { ENT-HN cancer } \\
\text { resection blocks }\end{array}$ & $\begin{array}{l}\text { ENT-HN cancer } \\
\text { resection slides }\end{array}$ & $\begin{array}{l}\text { Total histochemical } \\
\text { stains }\end{array}$ & Total immunostains & Oversize blocks \\
\hline 1998 & 1735 & 1914 & 600 & 615 & 337 & 322 & No data \\
\hline 1999 & 2075 & 2335 & 699 & 703 & 367 & 436 & 35 \\
\hline 2000 & 2160 & 2421 & 748 & 754 & 356 & 438 & 50 \\
\hline 2001 & 2724 & 3133 & 1020 & 1064 & 473 & 724 & 60 \\
\hline
\end{tabular}

ENT, ear, nose, and throat; $\mathrm{HN}$, head and neck. 
Table 3 Prospective study of 11 consecutive cancer resection specimens with time analysis

\begin{tabular}{lll}
\hline Task & Average & Range \\
\hline Prosection & 50 minutes & $15-95$ minutes \\
Microscopic examination & 47 minutes & $10-100$ minutes \\
Reporting and authorisation & 20 minutes & $2-45$ minutes \\
Blocks examined & 19.36 & $11-29$ \\
Technical input (Welcan & 283 units & $113-484$ units \\
units) & & \\
\hline
\end{tabular}

an average of 117 minutes of a pathologist's time, with a median time of 110 minutes. This included macroscopic and microscopic examination, with or without photography, and the issuing of a report. For each of the 11 cases, the time taken to examine each block was calculated (pathologists' time divided by the number of blocks examined). This ranged from 3.54 minutes/block to 7.58 minutes/block (average, 6.07 minutes/block). For these resections, the average technical input for each case was 283 Welcan units (range, 113-484 units; median, 264), which are comparable to units of time in minutes. This included calculations from specimen reception through to the generation of sections for reporting. Additional histochemical or immunohistochemical studies were not required, although oversize blocks were examined in seven cases.

Between 1998 and 2001, the number of histochemical procedures increased by $40 \%$ (337 to 473 ), but a more striking increase of $124 \%$ (332 to 724 ) was seen for immunohistochemical examinations.

To obtain the number of cytological and histological investigations undertaken during the management of one patient with cancer, a retrospective review of 15 of the 62 patients who underwent cancer resection in 2001 was carried out. All but one of the 15 patients underwent preoperative surgical biopsies. Fine needle aspiration was carried out in 10 patients, preoperatively in nine and after surgery in three. Only one type of investigation was carried out in six patients-five had surgical biopsies and one patient had fine needle aspiration (FNA). The maximum number of surgical biopsies carried out for each patient was nine and the number of FNAs was three. In all, 50 surgical biopsies and 16 FNAs were undertaken, giving an average of 3.3 biopsies (median, 2) and 1.06 FNAs (median, 1) for each patient. Of these, 44 histological biopsies and 11 FNAs were carried out before the cancer surgery. Non-diagnostic tests were not excluded from our analysis.

\section{DISCUSSION}

The workload in cellular pathology departments is increasing both in terms of quantity and the complexity of the examination procedure. However, there is little objective evidence of this increase, which has been brought about by changes in both clinical and pathological practice. Our study has sought to identify the alteration in the workload in a department of cellular pathology, generated by the ENT-HN surgical specialty. Changes within the clinical ENT-HN service in 1995 and the publication of a minimum dataset for head and neck cancer in 1998 provided benchmarks for gauging the change.

Between 1995 and 2001, the number of specimens generated by the ENT-HN unit at the Oxford Radcliffe Hospitals increased by $88.83 \%$, in contrast to a $13.57 \%$ increase in the total surgical pathology workload during the same period. This increase in the ENT-HN case workload can best be explained by the reorganisation of the ENT-HN clinical service. In 1995, the appointment of a specialist head and neck surgeon resulted in the formation of the HN oncology service within the ENT department of the trust. This is now a cancer centre and a tertiary referral centre, serving a community of 850000 . Three specialist HN surgeons now provide their services to this unit. This reconfiguration and expansion of service has clearly impacted on the ENT-HN workload of cellular pathology. Between 1997/98 and 2000/2001, the number of patients referred to the ENT department increased by $12.81 \%$, which resulted in a $36.57 \%$ increase in the ENT-HN histology workload over a corresponding, although not identical, period (1998-2001). This non-proportionate increase in histology workload probably reflects the increasingly specialist nature of the referrals, which require multidisciplinary management.

\section{"Increasingly, prognosis and further management of patients, especially of those with cancer, is dependent to a large extent on the histopathological report"}

Treatments are becoming more refined and complicated. The universal use of immunohistochemistry and the availability of molecular biological techniques have led throughout cellular pathology to the definition of disease subsets, recognition of new entities, and to the identification of proteins that serve as markers of prognosis (for example, HER2, oestrogen receptors, etc). Increasingly, prognosis and further management of patients, especially of those with cancer, is dependent to a large extent on the histopathological report. As a consequence, the amount of pathological information required by clinicians is increasing. In recognition of this need, the Royal College of Pathologists has issued minimum datasets for the examination and reporting of cancer resection specimens. This series of "Standards and minimum datasets for reporting common cancers" currently numbers 16 separate booklets and covers all major sites. The dataset for head and neck cancer was published in November 1998. This requires detailed documentation of tumour size, depth of invasion, relation to excision margins, invasion of vascular, neural and bony/cartilaginous structures, numbers and sizes of lymph nodes involved, and their levels and evidence of extracapsular spread. This has increased the complexity and labour intensity of the pathological analysis by necessitating the examination of additional blocks for different margins and of separate blocks for the various lymph node levels. Although a quantitative change in the workload is easy to assess, there is currently no accepted measure of the complexity of work undertaken in cellular pathology. This was acknowledged in the Royal College of Pathologists' publication on "Medical and scientific staffing of national health service pathology departments" in June $1999,{ }^{7}$ which provided guidance on the workload and staffing levels in laboratories. This recommended 4000 surgical requests for each whole time equivalent district general hospital pathologist and 2000 specimens for a pathologist in an academic department. These figures were published at a time when most pathologists were generalists, and were based on an average degree of case complexity for a typical department. The document acknowledged the difficulty in introducing weighting factors for more complicated specimens to increase the accuracy of workload assessment, and suggested lower numbers for departments with a more demanding case mix. It also suggested that a simpler way of adjusting workload measurements may be possible by counting the total number of microscopic slides to indicate the complexity of each case, but more data were required. 
There is now an urgent need for a composite measure(s) of the workload, given the increasing subspecialisation in cellular pathology.

One such measure of complexity is the number of paraffin wax blocks taken and slides examined during the reporting of a specimen, which is a direct measure of the work intensity. Between 1998 and 2001, the number of blocks and slides examined for all ENT-HN cases increased by 57\% and $63.7 \%$, respectively. This change was more than could be accounted for by the $36.57 \%$ increase in specimen numbers alone. When viewed for cancer resection specimens, the change was more striking. The total number of blocks and slides increased by $70 \%$ and $73 \%$, respectively, and the number of blocks and slides examined for each case increased by $50.9 \%$ (10.9 to 16.45 ) and $53 \%$ (11.18 to 17.16), respectively. This should be compared with a $12.72 \%$ (55 to 62) increase in the actual number of resections. When large resections were excluded, the increase for other ENTHN cases was a more modest $8 \%$ (2.05 to 2.22$)$ for blocks and $14 \%$ (2.35 to 2.69) for slides for each case, respectively. These figures demonstrate the upward trend in the number of blocks and slides processed and examined, providing firm evidence of an increase in detail and complexity of the examination procedure. This unit of "blocks and/or slides for each case" is an accurate and objective measure of complexity, which can be applied across the different subspecialties in pathology (see later).

Our study found that on average the complete examination of one ENT-HN cancer resection took 117 minutes of a pathologist's time. These calculations are a minimal estimation because no consideration was made for clinicopathological and multidisciplinary meetings integral to the cancer services. In terms of a notional session of 210 minutes, 117 minutes accounts for over half $(55 \%)$ a session.

Increases of $40 \%$ and $124 \%$ in the number of histochemical and immunohistochemical requests illustrate an increasing complexity of histopathological diagnosis beyond routine haematoxylin and eosin stained sections. A possible explanation for the increase of $124 \%$ is that immunohistochemistry is routinely required in classifying lymphomas on lymph node biopsies. Other areas include subtyping variants of squamous cell carcinoma and uncommon salivary gland neoplasms.

These figures clearly demonstrate the increase in workload, both in quantity and complexity, which can be correlated to the clinical service reorganisation. How can any of these indicators be used for measuring workload in cellular pathology? Pegging case load to clinical sessions could be one way of achieving this under the current contractual agreements. For clinical specialties, individual consultant workloads are based on sessional commitments that are compartmentalised into ward rounds, outpatient clinics, operating lists, etc. Workload is related to a fixed number (six or seven) of clinical sessions with protected time for audit, research, and management. Although clinicians are able to manage their workload effectively by limiting the number of patients seen to clinically reasonable levels, this is not the case in cellular pathology, where there is no inherent control over the workload. The methodology used in the prospective analysis of 11 cancer resection cases in our study can be used to calculate the number of (any type of) specimens reportable in one session, and a projection made of an acceptable annual workload. This assessment of workload is best individualised to reflect upon the local pathology practice and degree(s) of subspecialisation. Using the cut up as a starting point, the time to report cases (cut up, microscopy, reporting, and checking) can be recorded. When done over different time periods, it would cover the case mix encountered and the special interests of the pathologist. From this, an average estimate of the number of cases reportable in one "clinical session" can be calculated. As mentioned previously, this does not include participation in multidisciplinary meetings, an essential part of the workload. Confidence in this methodology will prevail when there is no undue introduction of time elements to "err on the side of caution".

The number of blocks and/or slides for each case is a useful measure of complexity, but there are two factors that preclude the definition of a "one size fits all" indicator or a rigidly defined figure for an indicator to measure workload. One is the differing practice of histology in departments across the country, with varying degrees of specialisation in place. The other factor is a variation in case mix, not just among different organ systems, but also within them, which results in variable case complexity. This is demonstrated by the prospective analysis of 11 resection cases of ENT-HN cancer, where the number of blocks examined for each case ranged from 11 to 29. When the pathologists' time was divided by the total number of blocks examined, a range of 3.54-7.58 minutes was obtained, indicating that some cases took longer to report than others.

In the cellular pathology department of the Oxford Radcliffe Hospitals, the management of one patient with ENT-HN cancer currently generates an average of 3.3 biopsies and 1.06 cytological specimens. Calculating the number of investigations involved in the management of patients with cancer can help plan an adequately staffed service by supplying an approximate projection of the workload, based on population statistics.

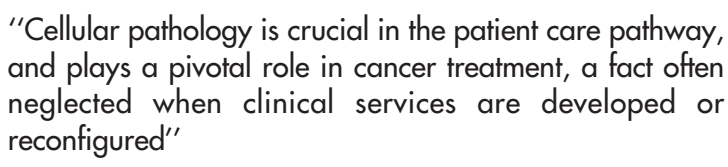

The examination of one cancer resection took an average of 283 minutes (Welcan units) of technician's time. Within a working week of 37 hours, this occupies over a 10th of one technician's time. For any cellular pathology laboratory, ENTHN cancer constitutes one of the lower specimen loads when compared with that from other sources, such as gynaecology or gastroenterology. From our study, it is apparent that the current reporting practice has a substantial impact on technical staffing needs. This is a reminder that clinical service reorganisation affects the workload not only of the medical, but also the biomedical, staff. The existing management practice assesses the appointment of new clinical consultants by its impact on the medical staffing of a pathology department. Hardly ever is attention paid to the biomedical workforce, which is facing a similar, if not a more profound shortage. In our study, the impact of increases in the workload on secretarial/administrative staff was not assessed. It is reasonable to assume that this would increase in direct proportion to the workload.

Cellular pathology is crucial in the patient care pathway, and plays a pivotal role in cancer treatment, a fact often neglected when clinical services are developed or reconfigured. Such manoeuvres increase departmental workloads and can lead to the provision of a suboptimal service. Consequently, there is pressure on cellular pathologists to subspecialise, although few centres in the UK have to date gone down this path completely, with most departments offering a "lead" pathologist who also participates in a general rota. The current 16 minimum datasets are sure to increase in number, requiring pathologists to provide detailed reports, thereby increasing their workloads, often with no additional medical and technical support. The recognition of cellular pathology as a clinical rather than a technical service 


\section{Take home messages}

- Between 1995 and 2001, as a result of clinical service reconfiguration in our ear, nose, and throat (ENT), head and neck (HN) department there has been an increase both in cellular pathology workload and in its complexity

- There was an $88.83 \%$ increase in ENT-HN specimens but only a $13.53 \%$ increase in total surgical workload

- Work complexity was measured as blocks/slides for each case and number of histochemical/immunohistochemical requests, objective measures that are applicable to all pathology subspecialties

- Given the workforce crisis and expanding clinical needs, realistic workload calculations should include the measurement of complexity and not just volumes

is long overdue, and one way of alleviating the medical recruitment crisis in this profession would be by tackling the issues of workload and clinical recognition.

The 1999 Royal College of Pathologists' recommendations of 4000 requests/district general hospital pathologist and 2000/academic pathologist now need careful reappraisal. Strict adherence by management to these "guidelines" would result in unrealistic staffing levels to meet today's workload and clinical needs. It is our recommendation that departments handling large, complicated pathological specimens reassess their workload to staffing ratio based on the complexity of the work undertaken. Our study has not provided a "magic bullet" or easy solution to tackling the workload assessment problem, because there probably is none. It has suggested ways of measurement that reflect upon the current practice and would need revising when that changes significantly. This is certainly not the only or possibly the best way of workload assessment, but our study has documented that it can be achieved objectively in a manner that deals with both its quantitative and qualitative components.

\section{NOTE IN PROOF}

The revised "Guidelines on staffing and workload for histopathology and cytopathology departments" (The Royal College of Pathologists, July 2003) provides a framework for assessing the complexity of work, in addition to its volume. This has addressed one concern raised in our paper on the usefulness of the 1999 guidance in light of current practice. Our study has suggested assessment criteria that can be used to provide an objective and comprehensive assessment of workloads.

\section{Authors' affiliations}

S Winter, G J Cox, R Corbridge, Department of ENT and Head and Neck Surgery, The Raddliffe Infirmary, Woodstock Road, Oxford OX2 6HE, UK

A J Chaplin, P R Millard, K A Shah, Department of Cellular Pathology, The John Raddliffe Hospital, Headington, Oxford OX3 9DU, UK

\section{REFERENCES}

1 A policy framework for commissioning cancer services. A report by the expert advisory group of cancer to the Chief Medical Officer of England and Wales, April 1995.

2 Provision and quality assurance for head and neck cancer in the UK. London: The British Association of Head and Neck Oncologists, 1998.

3 Effective head and neck cancer management, a consensus document. London: The British Association of Otolaryngologists, Head and Neck Surgeons, 1998.

4 The NHS cancer plan. London: Department of Health, September, 2000.

5 WELCAN UK. Workload measurement system manual, $1992 \mathrm{ed}$. West Glamorgan, UK: WELCAN UK.

6 Minimum dataset for head and neck carcinoma histopathology reports. Standards and minimum datasets for reporting common cancer. London: The Royal College of Pathologists, November 1998.

7 Medical and scientific staffing of National Health Service pathology departments. London: The Royal College of Pathologists, June 1999. 